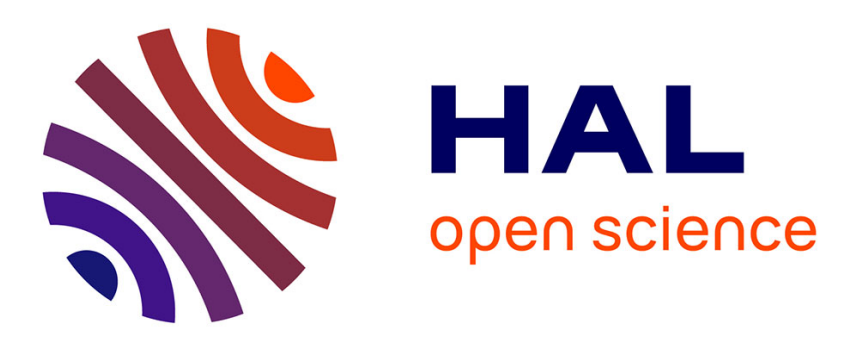

\title{
How to deal with point correspondences and tangential velocities in the level set framework
}

Jean-Philippe Pons, Gerardo Hermosillo, Renaud Keriven, Olivier Faugeras

\section{To cite this version:}

Jean-Philippe Pons, Gerardo Hermosillo, Renaud Keriven, Olivier Faugeras. How to deal with point correspondences and tangential velocities in the level set framework. RR-4857, INRIA. 2003. inria00071726

\section{HAL Id: inria-00071726 \\ https://hal.inria.fr/inria-00071726}

Submitted on 23 May 2006

HAL is a multi-disciplinary open access archive for the deposit and dissemination of scientific research documents, whether they are published or not. The documents may come from teaching and research institutions in France or abroad, or from public or private research centers.
L'archive ouverte pluridisciplinaire HAL, est destinée au dépôt et à la diffusion de documents scientifiques de niveau recherche, publiés ou non, émanant des établissements d'enseignement et de recherche français ou étrangers, des laboratoires publics ou privés. 
INSTITUT NATIONAL DE RECHERCHE EN INFORMATIQUE ET EN AUTOMATIQUE

\section{How to deal with point correspondences and tangential velocities in the level set framework}

Jean-Philippe Pons — Gerardo Hermosillo — Renaud Keriven — Olivier Faugeras

$\mathbf{N}^{\circ} 4857$

Juin 2003

THÈME 3 



\title{
How to deal with point correspondences and tangential velocities in the level set framework
}

\author{
Jean-Philippe Pons, Gerardo Hermosillo, Renaud Keriven, Olivier Faugeras \\ Thème 3 - Interaction homme-machine, \\ images, données, connaissances \\ Projet Odyssée \\ Rapport de recherche $n^{\circ} 4857$ - Juin 2003 - 21 pages
}

\begin{abstract}
In this report, we overcome a major drawback of the level set framework: the lack of point correspondences. We maintain explicit backward correspondences from the evolving interface to the initial one by advecting the initial point coordinates with the same velocity as the level set function. Our method leads to a system of coupled Eulerian partial differential equations. We show in a variety of numerical experiments that it can handle both normal and tangential velocities, large deformations, shocks, rarefactions and topological changes. Applications are many since our method can upgrade virtually any level set evolution. We complement our work with the design of non zero tangential velocities that preserve the relative area of interface patches; this feature may be crucial in such applications as computational geometry, grid generation or unfolding of the organs' surfaces, e.g. brain, in medical imaging. This report also tackles a diffeomorphic approach to level set evolution, a family of volume-preserving smoothing flows, and some numerical aspects of the intrinsic heat flow on implicit surfaces.
\end{abstract}

Key-words: level sets, point correspondences, diffeomorphisms, area preservation, cortex unfolding, mean curvature flow, surface diffusion flow, intrinsic heat flow, Laplace-Beltrami operator 


\section{Suivi de points et vitesses tangentielles avec la méthode des ensembles de niveaux}

Résumé : Dans ce rapport nous venons à bout d'une des limitations principales de la méthode des ensembles de niveaux : l'absence de correspondances point à point. Nous maintenons les correspondances explicites entre l'interface en mouvement et l'interface initiale en transportant les coordonnées initiales des points avec la même vitesse que la fonction de niveau. Notre méthode débouche sur un système couplé d'équations aux dérivées partielles Eulériennes. Nous montrons à travers de nombreux exemples qu'elle peut gérer les vitesses normales et tangentielles, les grandes déformations, les chocs, les raréfactions et les changements de topologie. Les applications sont innombrables étant donné que notre méthode peut se greffer sur n'importe quelle évolution de front par ensembles de niveaux. En complément de ce travail, nous décrivons comment calculer des vitesses tangentielles qui préservent l'aire relative des fragments de l'interface ; cette propriété peut être cruciale dans certaines applications comme la géométrie computationnelle, la génération de grille ou le dépliement de la surface des organes, par exemple du cerveau, en imagerie médicale. Ce rapport évoque également une approche difféomorphique de l'évolution de fronts par ensembles de niveaux, une famille de flots régularisants avec préservation du volume, et quelques aspects numériques de l'équation de la chaleur intrinsèque sur une surface implicite.

Mots-clés : ensembles de niveaux, correspondances point à point, difféomorphismes, préservation de l'aire, dépliement de cortex, flot par courbure moyenne, flot de diffusion de surface, équation de la chaleur intrinsèque, opérateur de Laplace-Beltrami 


\section{Contents}

1 Introduction $\quad 4$

2 Point correspondences in the level set framework 4

2.1 Method 1: From region tracking to general interface data . . . . . . . . 4

2.2 Method 2: Explicit backward point correspondences . . . . . . . . . 6

2.3 Numerical experiments . . . . . . . . . . . . . . . . . 7

3 Diffeomorphic level set evolution $\quad 11$

4 Relative area preserving tangential velocities $\quad 11$

4.1 Differential geometry formulation . . . . . . . . . . . . . . . . 12

4.2 Numerical experiments . . . . . . . . . . . . . . . . . 12

5 Conclusion $\quad 15$

$\begin{array}{ll}\text { A Intrinsic geometry } & 15\end{array}$

B Filling the gap between the averaged mean curvature flow and the surface diffusion flow $\quad 15$

C Some numerical aspects of the intrinsic heat flow on implicit surfaces 16

C.1 Discretization of the Laplace-Beltrami operator . . . . . . . . . . . 16

C.2 An additional extension term . . . . . . . . . . . . . . 18 


\section{Introduction}

One major drawback of the level set method [21] is the inability to keep point correspondences during the deformation. As a consequence it is not possible to handle data associated with a moving interface with the straightforward level set approach. Some hybrid Lagrangian-Eulerian methods have been proposed to circumvent this limitation [12, 25, 27]. However, for sake of stability and topology independence, a completely Eulerian approach would be desirable. In [5] the authors come to this end in the particular case of region tracking. In Section 2 we go one step further and design a new method that can handle general interface data and maintain explicit point correspondences during the evolution.

In Section 3 we outline a diffeomorphic approach to level set evolution in the case of smooth velocities.

We then turn to the design of non trivial (i.e. non zero) tangential velocities. This problem has been overlooked so far because it is known that tangential velocities have no influence on the geometry of the interface. But they do affect point correspondences. In Section 4 we show that in some cases interface data may be considerably altered without an adequate tangential velocity and we describe a method to build a tangential velocity that preserves the relative area of interface patches, i.e. the ratio between the area of any patch and the total area of the interface. Our method can be an alternative to the area preserving mappings worked out in [1] for the purpose of cortex unfolding and virtual colonoscopy.

In Appendix A we recall the results of intrinsic differential geometry that are used in the text. In Appendix B we propose a one-parameter family of volume-preserving smoothing flows which fills the gap between the averaged mean curvature flow and the surface diffusion flow.

Finally, in Appendix $\mathrm{C}$ we deal with some numerical aspects of the intrinsic heat flow on implicit surfaces.

\section{Point correspondences in the level set framework}

\subsection{Method 1: From region tracking to general interface data}

In [5], region tracking on a deforming interface $\Gamma$ is achieved in the level set framework by representing the boundary of the region of interest as the intersection of $\Gamma$ with an auxiliary surface $\hat{\Gamma}$. $\Gamma$ and $\hat{\Gamma}$ are represented as the zero level sets of the functions $\phi$ and $\hat{\phi}$ respectively. The latter are evolved according to the following system of coupled PDE's:

$$
\left\{\begin{array}{l}
\frac{\partial \phi}{\partial t}=\beta\|\nabla \phi\| \\
\frac{\partial \hat{\phi}}{\partial t}=\left(\beta \frac{\nabla \phi}{\|\nabla \phi\|} \cdot \frac{\nabla \hat{\phi}}{\|\nabla \hat{\phi}\|}\right)\|\nabla \hat{\phi}\|
\end{array}\right.
$$


where $\beta$ is the magnitude of the inward normal velocity of the interface $\Gamma$. Equation (1) also writes

$$
\left\{\begin{array}{l}
\mathbf{v}=-\beta \frac{\nabla \phi}{\|\nabla \phi\|} \\
\frac{\partial \phi}{\partial t}+\mathbf{v} \cdot \nabla \phi=0 \\
\frac{\partial \hat{\phi}}{\partial t}+\mathbf{v} \cdot \nabla \hat{\phi}=0 .
\end{array}\right.
$$

In equation (2) we can now clearly see that $\Gamma$ and $\hat{\Gamma}$ move with the same velocity $\mathbf{v}$. In [5] this velocity is taken normal to the interface but we could as well consider velocities containing a tangential part. Tangential velocities do not affect the evolution of the interface, but they do affect the evolution of $\hat{\Gamma}$ and hence of the region of interest.

Moreover, we notice that the zero level set of $\hat{\phi}$ does not play a particular role. All the level sets of $\hat{\phi}$ evolve according to v. Actually, the evolution equation for $\hat{\phi}$ is nothing but an advection equation with an extrinsic velocity field.

Hence we can safely forget that $\hat{\phi}$ stands for the level set function of a hypersurface and change it to a general scalar or vector-valued function $u$ coding for some data on the interface. For example, if we want to visualize neural activity on the cerebral cortex, $u$ could be the functional MRI signal. We then obtain a straightforward method to associate a quantity to a moving interface in the level set framework. This method is summarized by the following system of coupled PDE's:

$$
\left\{\begin{array}{l}
\frac{\partial \phi}{\partial t}+\mathbf{v} \cdot \nabla \phi=0 \\
\frac{\partial u}{\partial t}+\mathbf{v} \cdot \nabla u=0
\end{array}\right.
$$

where $\mathbf{v}$ is either given or defined from geometric properties of the zero level set of $\phi$ (e.g. normal, curvature, etc.).

In [28], an augmentation of this method is worked out to model the evolution of the concentration of surfactants along a moving interface in the level set framework: besides being advected, interface data is also evolved according to an intrinsic PDE along the interface. This method translates into a system of coupled PDE's where the evolution equation for the concentration is a convection diffusion equation which requires semi-implicit time stepping. Although more elaborate than equation (3), this formulation is not relevant to our problem of point correspondences.

Note that the method stated in equations (3) can be plugged into an existing level set interface evolution without any modification. Moreover, while the proper numerical scheme for equation (3a) depends on the chosen velocity, equation (3b) only requires a simple upwinding scheme since $\mathbf{v}$ is extrinsic with respect to $u$.

In many real problems, both $\mathbf{v}$ and $u$ have natural extensions off the interface. For instance, the fMRI signal is known throughout the scanning volume. Otherwise one may build an extension of $\mathbf{v}$ and/or $u$ that is constant along the normal to the interface by

$\mathrm{RR} \mathrm{n}^{\circ} 4857$ 
considering the steady state of the following PDE [4]:

$$
\frac{\partial u}{\partial t}+\operatorname{sign}(\phi)(\nabla u \cdot \nabla \phi)=0
$$

\subsection{Method 2: Explicit backward point correspondences}

Method 1 suffers from several limitations: it does not provide explicit point correspondences and requires to solve a PDE for each scalar component of the interface data.

In order to solve these problems we focus on the initial coordinates of the points of the interface. We regard the latter as vector-valued interface data that we can advect as suggested previously. Let us consider a function $\Psi: \mathbb{R}^{n} \times \mathbb{R}^{+} \mapsto \mathbb{R}^{n}$ such that

$$
\left\{\begin{array}{l}
\Psi(\mathbf{x}, 0)=\mathbf{x}, \\
\frac{\partial \Psi}{\partial t}+D \Psi \mathbf{v}=0,
\end{array}\right.
$$

where $D \Psi$ stands for the jacobian matrix of $\Psi$ with respect to the first $n$ variables.

For each point $\mathrm{x}$ of the interface at time $t, \Psi(\mathrm{x}, t)$ holds the position that this point occupied at time $t=0$. Hence $\Psi(., t)$ provides explicit backward correpondences from the current interface to the initial one.

Moreover, we can ignore equation (3b) and build any interface data by composition of some initial data $u_{0}$ with $\Psi$. Indeed, $u=u_{0} \circ \Psi$ formally satisfies equation (3b):

$$
\frac{\partial u}{\partial t}+\nabla u \cdot \mathbf{v}=\left(\nabla u_{0} \circ \Psi\right) \cdot\left(\frac{\partial \Psi}{\partial t}+D \Psi \mathbf{v}\right)=0 .
$$

We might be tempted to consider forward correspondences too by the means of a function $\hat{\Psi}: \mathbb{R}^{n} \times \mathbb{R}^{+} \mapsto \mathbb{R}^{n}$ such that

$$
\left\{\begin{array}{l}
\hat{\Psi}(\mathbf{x}, 0)=\mathbf{x}, \\
\frac{\partial \hat{\Psi}}{\partial t}=\mathbf{v} \circ \hat{\Psi} .
\end{array}\right.
$$

This approach is used in [25] to construct transverse lines for the purpose of grid generation with level set methods and in [27] for image registration. But the Eulerian PDE (4) has two important advantages over the Lagrangian ODE (5). First, it is numerically more stable since the computations are performed on a fixed grid. More importantly, it performs automatic deleting of merging characteristics, whereas this task requires intricate delooping algorithms in the Lagrangian approach.

Moreover, forward correspondences may not exist if the interface evolution forms shocks; the interface may even collapse and merely disappear. In such cases, equation (5) is not relevant.

For all the reasons discussed above, our method focuses on backward correspondences. In some applications, such as texture mapping or tracking of isolated points, forward correspondences are needed and it is necessary to invert the $\Psi$ map at a postprocessing stage.

INRIA 
If $\mathbf{v}$ is sufficiently smooth $[9,26]$, equation (4) generates a one-parameter family of diffeomorphisms. So does equation (5) and by the chain rule we get

$$
\Psi(., t) \circ \hat{\Psi}(., t)=\hat{\Psi}(., t) \circ \Psi(., t)=i d \forall t .
$$

This is not true for general velocities. Typically, $\Psi$ fails to be surjective in the presence of a shock and fails to be injective in the presence of a rarefaction.

\subsection{Numerical experiments}

For equations (3b) and (4) we use a simple upwind scheme, since the velocity is extrinsic to $u$ and $\Psi$. The proper scheme for equation (3a) depends on velocity properties; all the schemes needed in our experiments are taken from $[20,25]$. To estimate one-sided space derivatives we adopt the fifth order weigthed essentially non-oscillatory (WENO) scheme derived in $[14,13]$. As regards time differencing, a simple Euler scheme has proven sufficient in our experiments. For sake of efficiency we use the PDE-based fast local level set implementation proposed in [23].

Figure 1 demonstrates our method in several 2D test cases. Throughout this series of experiments, we use a 128x128 computational grid. All curves are extracted with a marchingcube algorithm [16] and the data are interpolated at resulting vertices. In all rows, the first column shows the initial curve painted with a texture $u$; the latter purposely holds both smooth parts and discontinuities. The second column shows the curve after deformation painted with the data tracked with method $1(\S 2.1)$. The two remaining columns show the results of method $2(\S 2.2)$ : the third column shows the final interface painted with the transformed texture $u_{0} \circ \Psi$; in the fourth column, $\Psi$ is plotted using cubic spline interpolation at one out of ten vertices. The arrow tails indicate the positions where $\Psi$ is evaluated and the arrow heads point to the corresponding values of $\Psi$. The initial and the final curves are plotted too as a dotted and a dashed line, respectively.

The first row shows a circle shrinking with a constant velocity in its normal direction and simultaneously advected by an extrinsic rigid rotation velocity field; this example demonstrates that both normal and tangential velocities can be implemented. The second and the third rows are dedicated to shocks and rarefactions; they present a shrinking and an expanding square respectively. The fourth row focuses on the merging of two expanding circles; this example demonstrates that our methods naturally handle topological changes. The fifth row depicts the evolution of a circle under a non-constant vorticity velocity field defined by

$$
\mathbf{v}(x, y)=\left(\begin{array}{l}
\left.\sin ^{2}(\pi x) \sin (2 \pi y)\right) \\
-\sin (2 \pi x) \sin ^{2}(\pi y)
\end{array}\right) .
$$

This example is more challenging than the previous ones because the flow considerably stretches the interface. The construction of an extension velocity constant along the normal to the interface has proven necessary here.

Our results are in remarkable agreement with the expected solutions. A comparison between columns 2 and 3 reveals that methods 1 and 2 perform similarly. However, in the 

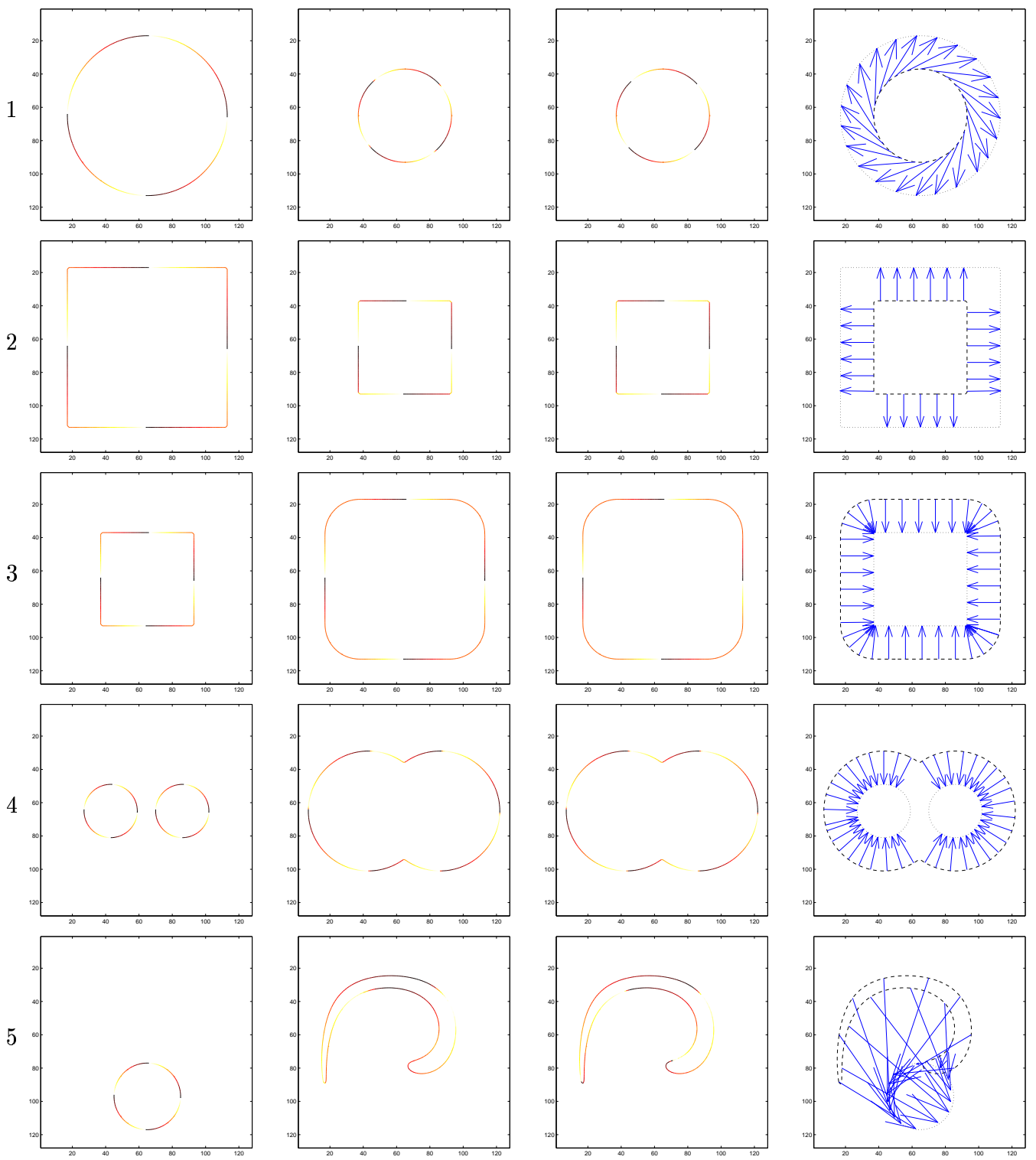

Figure 1: 2D examples of point correspondences in the level set framework (see text).

last row, we note that method 2 better preserves interface data discontinuities. A detailed view of these differences is diplayed in Figure 2(a,b). The latter method also has wider 
applications since it provides explicit point correspondences. Hence method 2 should be generally preferred to method 1 .

If we examine column 4 we note that the values of $\Psi$ on the final curves point almost exactly to the initial curves. In other words, although not enforced explicitely, the equality

$$
\phi=\phi_{0} \circ \Psi
$$

holds with a good accuracy. For further accuracy, it is possible to postprocess $\Psi$ as follows:

$$
\Psi \leftarrow \Psi+\left(\phi-\phi_{0} \circ \Psi\right) \frac{\nabla \phi_{0} \circ \Psi}{\left\|\nabla \phi_{0} \circ \Psi\right\|^{2}} .
$$

This correction helps to enforce equality (6) by projecting each value of $\Psi$ onto the suitable level set of $\phi_{0}$.

Figure 2(c,d) provides some detailed views of examples 2 and 4 , with a denser representation for $\Psi$. Arrow heads are hidden for better visualization. We can see that the expected discontinuities of $\Psi$ are successfully recovered.

We now focus on method 2 and three dimensions. In Figure 3 we use our method to unfold a cortex surface, extracted from MRI data of the human brain, with the averaged mean curvature flow; the displayed data are the mean curvature of the initial surface.

In order to test the robustness of our method to large deformations, we consider the following incompressible flow field proposed by LeVeque [15]:

$$
\mathbf{v}(x, y, z, t)=\cos \frac{\pi t}{T}\left(\begin{array}{c}
2 \sin ^{2}(\pi x) \sin (2 \pi y) \sin (2 \pi z) \\
-\sin (2 \pi x) \sin ^{2}(\pi y) \sin (2 \pi z) \\
-\sin (2 \pi x) \sin (2 \pi y) \sin ^{2}(\pi z)
\end{array}\right) .
$$

This flow is a surimposition of a deformation in the $x y$ plane with a deformation in the $x z$ plane. It reverses at time $T / 2$ so that the initial interface and data should be revovered at time $T$. This trick provides a convenient way to evaluate the correctness of our results. We take $T=1$ and we chose the plane $x=0.5$ as the initial interface. For our computations we use a $128 \times 128 \times 128$ computational grid.

Figure 4 shows the computed solution at $t=0,0.1,0.2,0.5,0.85$ and 1 . Rather than plotting point correspondences which are somewhat difficult to visualize in a 3D setting, we color the interface with some texture obtained by composition with $\Psi$. The mean square error on correspondences at $t=1$ is 0.16 voxels. Visually, the result is close to perfect. 


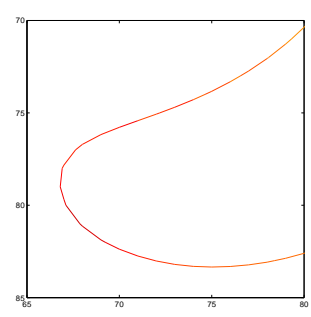

(a)

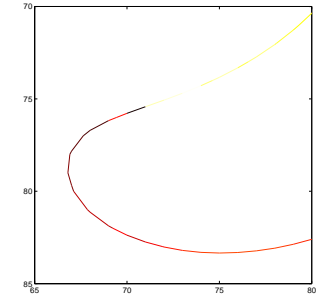

(b)

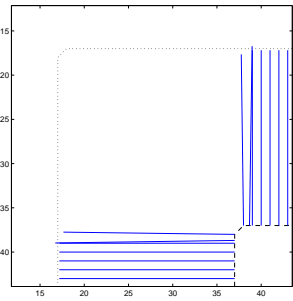

(c)

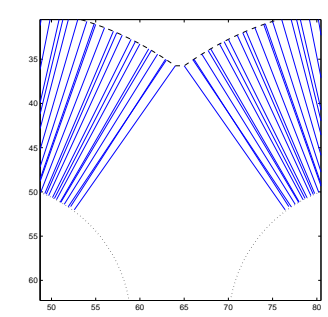

(d)

Figure 2: Detailed views of some parts of Figure 1.
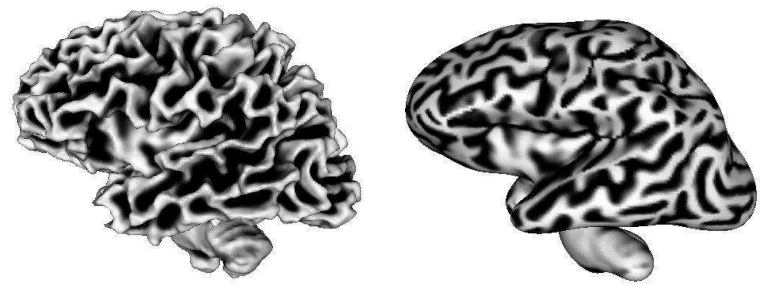

Figure 3: Cortex unfolding with method 2.

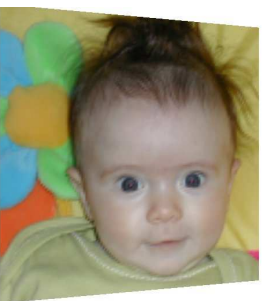

$\mathrm{t}=0$

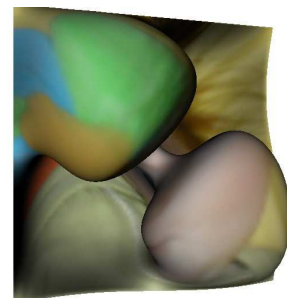

$\mathrm{t}=0.5$

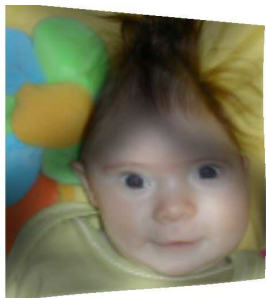

$\mathrm{t}=0.1$

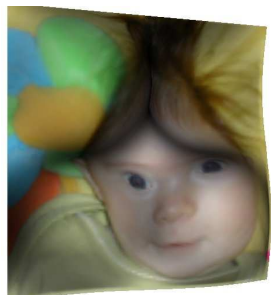

$\mathrm{t}=0.85$

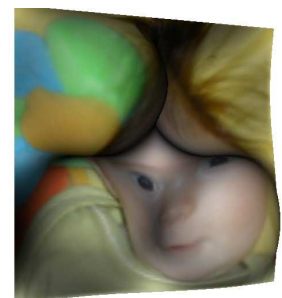

$\mathrm{t}=0.2$

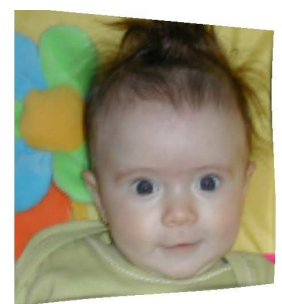

$\mathrm{t}=1$

Figure 4: 3D deformation test for method 2 (see text). 


\section{Diffeomorphic level set evolution}

As mentioned previously, for sufficiently smooth velocities $[9,26]$, equation (4) generates a one-parameter family of diffeomorphisms. A convenient way to satisfy the regularity requirements for $\mathbf{v}$ is to apply a Gaussian kernel of standard deviation $\sigma$ to the primary velocity field $\tilde{\mathbf{v}}$ prescribed by the (e.g. segmentation) problem: $\mathbf{v}=G_{\sigma} \star \tilde{\mathbf{v}}$. The resulting vector field is infinitely differentiable $\left(C^{\infty}\right)$. This smoothing step may replace the mean curvature term typically used to regularize surface evolutions.

In this case, the level set function can be readily obtained at all time by composition of the initial level set function $\phi_{0}$ with $\Psi$. Consequently, equation (3a) can be dropped and the whole evolution is summarized by equation (4) where $\mathbf{v}$ is now defined from $\phi_{0} \circ \Psi$. This method is very similar to the diffeomorphic image registration method formulated in [6]. As mentioned therein, a right composition update

$$
\Psi_{k+1} \leftarrow \Psi_{k} \circ\left(\mathrm{id}+\epsilon_{k} \mathbf{v}_{\mathbf{k}}\right)
$$

can then be choosen as a consistent, first order accurate, step forward operator for the approximation of equation (4).

Moreover, the moving interface is diffeomorphic to the initial one at all time, and the evolution preserves topology, which may be of great interest in applications where the desired topology is known, such as cortical surface reconstruction.

In [11] the authors achieve topology preservation in the level set framework by applying concepts from digital topology. Their method is based upon a modified update procedure of the discrete level set function which discards discrete topological changes, whereas the evolution would not be topology-preserving in its continuous formulation. On one hand, we object that such discrete methods lack theoretical justifications. On the other hand, they could be used as a complement of well-behaved, topology-preserving continuous flows as described in this section. Indeed, in spite of our continuous topology-preserving constraint, changes in digital topology can still occur if the distance between two parts of the interface falls below the voxel size.

\section{Relative area preserving tangential velocities}

Tangential velocities have no effect on the shape of evolving interfaces, but they do affect point correspondence and hence the evolution of interface data. In most problems where interface data are considered, a tangential velocity is unambiguously prescribed. However, one may encounter problems where no such natural tangential velocity exist and where interface data or point correspondences are nonetheless needed. The usual choice in such cases is a null tangential velocity, but we will demonstrate on some numerical experiments that this choice may considerably alter the local properties of interface data.

In this section we present a method to build non-trivial tangential velocities that preserve the relative area of interface patches, i.e. the ratio between the area of any patch and the total area of the interface. 


\subsection{Differential geometry formulation}

In differential geometry terms $[8,7]$, the condition stated above writes

$$
\operatorname{div}_{\Gamma} \mathbf{v}=\overline{\operatorname{div}_{\Gamma} \mathbf{v}}
$$

where $\operatorname{div}_{\Gamma}$ is the intrinsic divergence operator on $\Gamma$, and ${ }^{-}$denotes the average of a quantity along $\Gamma$.

If we decompose $\mathbf{v}$ into its outward normal component $v_{N}$ and its tangential part $\mathbf{v}_{T}$, equation (8) becomes

$$
\operatorname{div}_{\Gamma} \mathbf{v}_{T}+(n-1) H v_{N}=(n-1) \overline{H v_{N}},
$$

where $H$ denotes the mean curvature of $\Gamma$. Note that if the normal velocity is such that the evolution preserves the total area of the interface, then the right-hand sides of equations (8) and (9) vanish.

For a plane curve $C$ embedded in $\mathbb{R}^{2}$ deforming with velocity $\mathbf{v}=\alpha \mathbf{T}+\beta \mathbf{N}$, the relative area preservation condition boils down to $\frac{\partial \alpha}{\partial s}+\kappa \beta=\overline{\kappa \beta}$, where $\kappa$ denotes the curvature of $C$. This particular case has been used (with the inward normal convention) in [18] in the Lagrangian curve evolution framework: the authors build a tangential velocity that achieves a uniform redistribution of grid points along the curve, in order to overcome numerical instabilities caused by merging of grid points or by formation of the so-called swallow tails.

In [19], the authors design tangential forces which tend to equalise the surface of the faces of an evolving 3D simplex mesh, in order to maintain its geometric quality. Their approach is successful but, in contrast with our work, it only has a discrete formulation and cannot be generalized to other types of deformable models.

We now outline our method. Given a normal velocity field $v_{N}$, let us consider the solution $\eta$ of the following intrinsic Poisson equation on $\Gamma$ :

$$
\Delta_{\Gamma} \eta=(n-1)\left(H v_{N}-\overline{H v_{N}}\right),
$$

where $\Delta_{\Gamma}$ denotes the Laplace-Beltrami operator on $\Gamma$. Finding a solution of equation (10) is possible because the right-hand side is of zero average and the existence of a solution in this case is proved in [24]. Moreover, the solution $\eta$ is only defined up to a constant.

Then it is clear that

$$
\mathbf{v}=v_{N} \mathbf{N}-\nabla_{\Gamma} \eta
$$

where $\nabla_{\Gamma}$ denotes the intrinsic gradient on $\Gamma$, verifies equation (9). Note that the normal velocity is not altered since $\nabla_{\Gamma} \eta$ is purely tangential, and that the resulting velocity is non local: it depends on the whole shape and motion of the interface.

\subsection{Numerical experiments}

For each time step we have to solve an intrinsic Poisson equation. This can be done with a finite element technique on a triangulation of the interface as in [2]. Equation (10) then 
translates into a linear system with a sparse symmetric positive semi-definite matrix suited for numerical iterative methods such as the conjugate gradient (CG) method [3].

However, this method is not natural in the level set framework: it requires to compute a new triangulation and to extend the computed tangential velocity off the interface for each time step. Hence we adopt a cartesian grid approach to solve the Poisson equation, based on the discretization of the Laplace-Beltrami operator proposed in [4]. We have computed explicitly a discretized Laplace-Beltrami operator in 2 and 3 space dimensions by averaging complementary schemes which alternate backward and forward differences for the computation of the gradient and the divergence. Following [23], we compute the average $\overline{H v_{N}}$ required in the right-hand side of the Poisson equation on the cartesian grid using a smoothed version of the Dirac function:

$$
\begin{gathered}
\overline{H v_{N}}=\frac{\int H v_{N} \delta(\phi)|\nabla \phi| d \mathbf{x}}{\int \delta(\phi)|\nabla \phi| d \mathbf{x}}, \\
\delta(\phi)= \begin{cases}\frac{1}{2 \epsilon}\left(1+\cos \left(\frac{\pi \phi}{\epsilon}\right)\right) & \text { if }|\phi|<\epsilon, \\
0 & \text { otherwise. }\end{cases}
\end{gathered}
$$

Equation (10) then translates into a linear system with a sparse symmetric indefinite matrix which we solve with a minimum residual (MINRES) method [3]. We use the solution $\eta$ of time step $t-1$ as the initial guess for the MINRES method at time step $t$. This way, solving the Poisson equation with the desired accuracy only requires a handful of iterations.

In Figure 5 we revisit the case of a shrinking or expanding square with a null tangential velocity (top) and with a relative area preserving tangential velocity computed with our method (bottom). In Figure 6 we detail the case of a free-hand folded figure evolving under the averaged mean curvature flow. This latter case illustrates that interface data may be considerably altered without an adequate tangential velocity. Indeed, note how the large interior part with high curvature of the initial curve (first column) turns into a much smaller patch on the final curve (columns 2 and 3, top row). The corresponding data are unacceptably distorted. We insist on the fact that this phenomenon is not an artefact of our point tracking method: it is intrinsic to the evolution. In contrast, in the second row, the data are uniformly redistributed along the curve as expected.

We finish with a cortex unfolding example in Figure 7. We simulate a tumor on the left temporal gyrus of a subsampled human brain (left). The area of the tumor is considerably underestimated if the cortex is unfolded with a regular mean curvature flow (middle). This does not occur if we use a relative area preserving tangential velocity (right). 

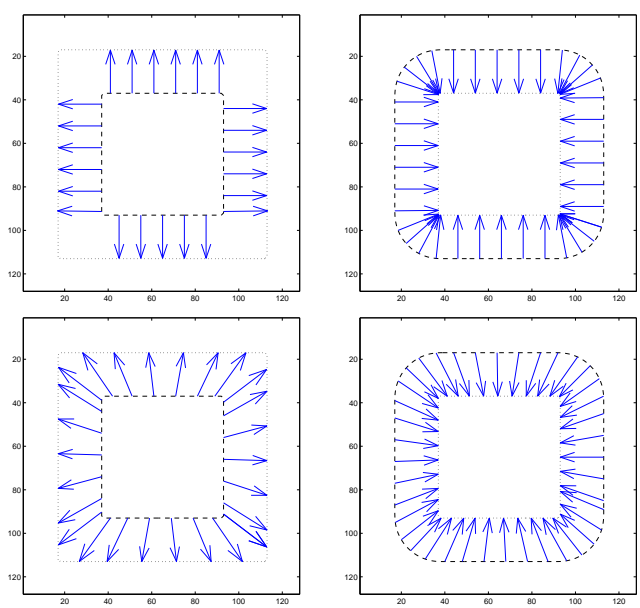

Figure 5: Some basic 2D evolutions with (bottom) and without (top) an area preserving tangential velocity.

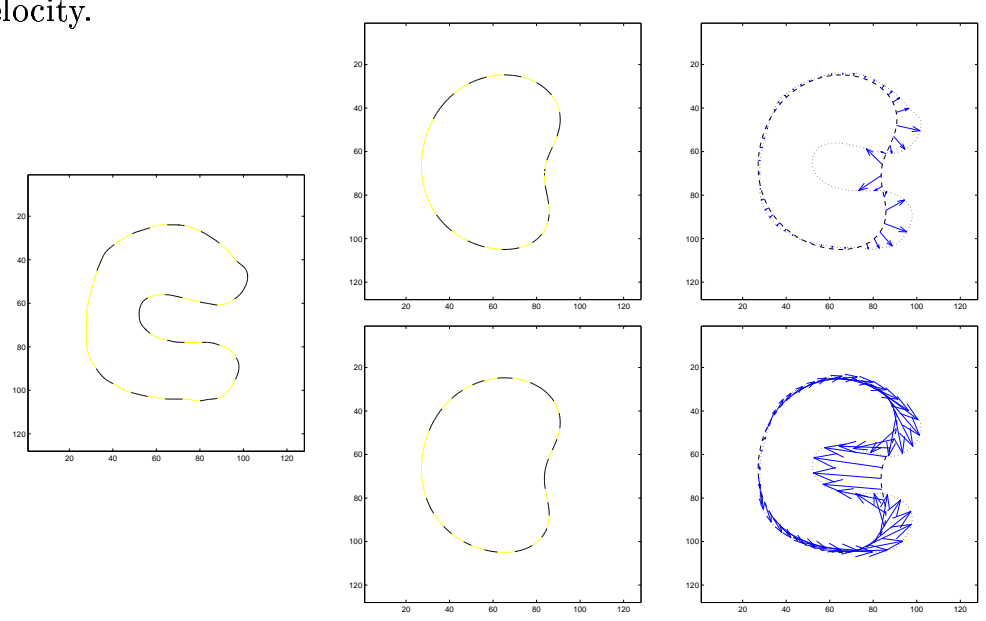

Figure 6: 2D unfolding with (bottom) and without (top) an area preserving tangential velocity.
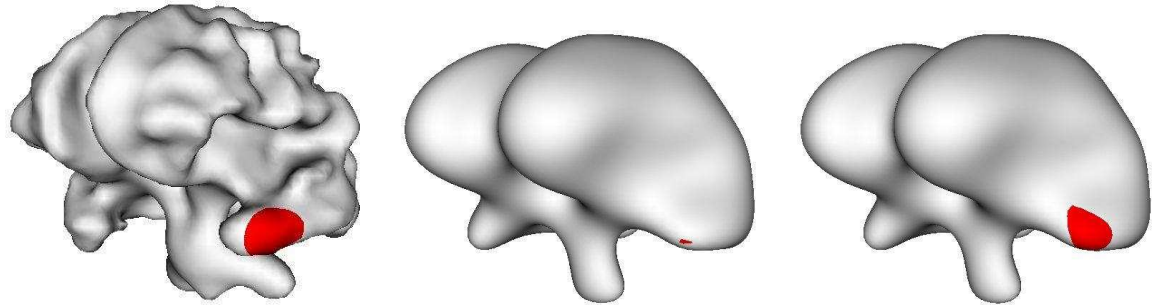

Figure 7: Cortex unfolding with (right) or without (middle) a relative area preserving tangential velocity.

INRIA 


\section{Conclusion}

As a conclusion, we have described a completely Eulerian method to maintain point correspondences in the level set framework. Our method is capable of handling both normal and tangential velocities, large deformations, shocks, rarefactions and topological changes. As an application of this idea, we have shown that a zero tangential velocity field (a normal flow) may unacceptably distort interface data in some cases. We have solved this problem by designing a relative area preserving tangential velocity which uniformly redistributes data along the interface.

\section{A Intrinsic geometry}

We recall in this appendix the few formulas of intrinsic differential geometry that are used in this report.

$$
\begin{aligned}
\Delta_{\Gamma} f & =\operatorname{div}_{\Gamma}\left(\nabla_{\Gamma} f\right) \\
\operatorname{div}_{\Gamma}(f \mathbf{v}) & =\nabla_{\Gamma} f \cdot \mathbf{v}+f \operatorname{div}_{\Gamma} \mathbf{v} \\
\operatorname{div}_{\Gamma} \mathbf{N} & =(n-1) H
\end{aligned}
$$

\section{B Filling the gap between the averaged mean curvature flow and the surface diffusion flow}

We propose a one-parameter family of volume-preserving smoothing flows that encompasses

two well-studied flows : the averaged mean curvature flow and the surface diffusion flow (see [17] and references therein) given in equations (15) and (16) respectively.

$$
\begin{aligned}
\mathbf{v} & =-(H-\bar{H}) \mathbf{N} \\
\mathbf{v} & =\Delta_{\Gamma} H \mathbf{N}
\end{aligned}
$$

In order to build our family of volume-preserving smoothing flows, we first define a linear smoothing operator on the interface. Let us consider the solution $u$ of the intrinsic heat equation on $\Gamma$ with initial condition $u_{0}$ :

$$
\left\{\begin{array}{l}
u(., 0)=u_{0} \\
\frac{\partial u}{\partial \tau}=\Delta_{\Gamma} u
\end{array} .\right.
$$

We then denote by $L_{\tau} u_{0}$ its solution $u(., \tau)$ at time $\tau>0$. With this notation in hand, our family of volume-preserving smoothing flows writes

$$
\mathbf{v}_{\tau}=-\left(H-L_{\tau} H\right) \mathbf{N} .
$$


Note that the time $\tau$ during which we diffuse the mean curvature $H$ of the interface $\Gamma$ is independent of the time $t$ that rules its evolution given by the equation $\frac{\partial \Gamma}{\partial t}=\mathbf{v}_{\tau}$.

These flows preserve the total volume enclosed by the interface since $\int_{\Gamma} \mathbf{v}_{\tau} \cdot \mathbf{N} d \mathbf{x}=0$. Indeed, by definition we have

$$
\frac{\partial\left(\int_{\Gamma} L_{\tau} H\right)}{\partial \tau}=\int_{\Gamma} \Delta_{\Gamma} H=0
$$

according to Appendix A. Therefore the integral $\int_{\Gamma} L_{\tau} H$ is constant and equal to its value for $\tau=0$. In addition, if $\Gamma$ is not connected, the volume of each connected component is preserved. At last, we have

$$
\begin{aligned}
\lim _{\tau \rightarrow+\infty} \mathbf{v}_{\tau} & =-(H-\bar{H}) \mathbf{N}, \\
\lim _{\tau \rightarrow 0+} \frac{\mathbf{v}_{\tau}}{\tau} & =\Delta_{\Gamma} H \mathbf{N} .
\end{aligned}
$$

where $\bar{H}$ is the average of $H$ in each connected component of $\Gamma$.

The first equation comes from the fact that the solution of the intrinsic heat equation on a connected compact manifold becomes constant when time $(\tau)$ goes to infinity and therefore equal to the mean of the initial condition.

The second equation comes from a semi-group property of the intrinsic heat equation operator (see for example $[22,10])$ :

$$
\lim _{\tau \rightarrow 0^{+}} \frac{L_{\tau}-H}{\tau}=\Delta_{\Gamma}
$$

\section{Some numerical aspects of the intrinsic heat flow on implicit surfaces}

\section{C.1 Discretization of the Laplace-Beltrami operator}

The Laplace-Beltrami operator of a function $u$ on a surface represented as the zero level set of $\phi$ writes

$$
\Delta_{\Gamma} u=\frac{1}{\|\nabla \phi\|} \nabla \cdot\left(P_{\nabla \phi} \nabla u\|\nabla \phi\|\right) .
$$

where $P_{\mathbf{v}}$ is the operator that projects a vector onto the hyperplane orthogonal to $\mathbf{v}$. Following [4], we have computed explicitly a discretized Laplace-Beltrami operator by averaging complementary schemes which alternate backward and forward differences for the computation of the gradient and the divergence. We have obtained the following expression in three space dimensions: 


$$
\begin{aligned}
& \mathbf{N}_{i, j, k}=\frac{1}{2}\left(\phi_{i+1, j, k}-\phi_{i-1, j, k}, \phi_{i, j+1, k}-\phi_{i, j-1, k}, \phi_{i, j, k+1}-\phi_{i, j, k-1}\right) \\
& c_{+x}=\left(\left\|\mathbf{N}_{i, j, k}\right\|-{N_{i, j, k}^{x}}^{2} /\left\|\mathbf{N}_{i, j, k}\right\|+\left\|\mathbf{N}_{i+1, j, k}\right\|-N_{i+1, j, k}^{x} /\left\|\mathbf{N}_{i+1, j, k}\right\|\right) / 2 \\
& c_{-x}=\left(\left\|\mathbf{N}_{i, j, k}\right\|-N_{i, j, k}^{x} /\left\|\mathbf{N}_{i, j, k}\right\|+\left\|\mathbf{N}_{i-1, j, k}\right\|-N_{i-1, j, k}^{x} /\left\|\mathbf{N}_{i-1, j, k}\right\|\right) / 2 \\
& c_{+y}=\left(\left\|\mathbf{N}_{i, j, k}\right\|-N_{i, j, k}^{y}{ }^{2} /\left\|\mathbf{N}_{i, j, k}\right\|+\left\|\mathbf{N}_{i, j+1, k}\right\|-N_{i, j+1, k}^{y}{ }^{2} /\left\|\mathbf{N}_{i, j+1, k}\right\|\right) / 2 \\
& c_{-y}=\left(\left\|\mathbf{N}_{i, j, k}\right\|-N_{i, j, k}^{y}{ }^{2} /\left\|\mathbf{N}_{i, j, k}\right\|+\left\|\mathbf{N}_{i, j-1, k}\right\|-N_{i, j-1, k}^{y} /\left\|\mathbf{N}_{i, j-1, k}\right\|\right) / 2 \\
& c_{+z}=\left(\left\|\mathbf{N}_{i, j, k}\right\|-N_{i, j, k}^{z} /\left\|\mathbf{N}_{i, j, k}\right\|+\left\|\mathbf{N}_{i, j, k+1}\right\|-N_{i, j, k+1}^{z}{ }^{2} /\left\|\mathbf{N}_{i, j, k+1}\right\|\right) / 2 \\
& c_{-z}=\left(\left\|\mathbf{N}_{i, j, k}\right\|-N_{i, j, k}^{z}{ }^{2} /\left\|\mathbf{N}_{i, j, k}\right\|+\left\|\mathbf{N}_{i, j, k-1}\right\|-N_{i, j, k-1}^{z}{ }^{2} /\left\|\mathbf{N}_{i, j, k-1}\right\|\right) / 2 \\
& c_{+x y}=N_{i+1, j, k}^{x} N_{i+1, j, k}^{y} /\left\|\mathbf{N}_{i+1, j, k}\right\| \quad c_{-x y}=N_{i-1, j, k}^{x} N_{i-1, j, k}^{y} /\left\|\mathbf{N}_{i-1, j, k}\right\| \\
& c_{+x z}=N_{i+1, j, k}^{x} N_{i+1, j, k}^{z} /\left\|\mathbf{N}_{i+1, j, k}\right\| \quad c_{-x z}=N_{i-1, j, k}^{x} N_{i-1, j, k}^{z} /\left\|\mathbf{N}_{i-1, j, k}\right\| \\
& c_{+y x}=N_{i, j+1, k}^{y} N_{i, j+1, k}^{x} /\left\|\mathbf{N}_{i, j+1, k}\right\| \quad c_{-y x}=N_{i, j-1, k}^{y} N_{i, j-1, k}^{x} /\left\|\mathbf{N}_{i, j-1, k}\right\| \\
& c_{+y z}=N_{i, j+1, k}^{y} N_{i, j+1, k}^{z} /\left\|\mathbf{N}_{i, j+1, k}\right\| \quad c_{-y z}=N_{i, j-1, k}^{y} N_{i, j-1, k}^{z} /\left\|\mathbf{N}_{i, j-1, k}\right\| \\
& c_{+z x}=N_{i, j, k+1}^{z} N_{i, j, k+1}^{x} /\left\|\mathbf{N}_{i, j, k+1}\right\| \quad c_{-z x}=N_{i, j, k-1}^{z} N_{i, j, k-1}^{x} /\left\|\mathbf{N}_{i, j, k-1}\right\| \\
& c_{+z y}=N_{i, j, k+1}^{z} N_{i, j, k+1}^{y} /\left\|\mathbf{N}_{i, j, k+1}\right\| \quad c_{-z y}=N_{i, j, k-1}^{z} N_{i, j, k-1}^{y} /\left\|\mathbf{N}_{i, j, k-1}\right\|
\end{aligned}
$$

$$
\begin{aligned}
\Delta_{\Gamma} u_{i, j, k}= & -\left(c_{+x}+c_{-x}+c_{+y}+c_{-y}+c_{+z}+c_{-z}\right) u_{i, j, k} \\
& +c_{+x} u_{i+1, j, k}+c_{-x} u_{i-1, j, k}+c_{+y} u_{i, j+1, k} \\
& +c_{-y} u_{i, j-1, k}+c_{+z} u_{i, j, k+1}+c_{-z} u_{i, j, k-1} \\
& -\left(c_{+x y}+c_{+y x}\right) u_{i+1, j+1, k}+\left(c_{-x y}+c_{+y x}\right) u_{i-1, j+1, k} \\
& +\left(c_{+x y}+c_{-y x}\right) u_{i+1, j-1, k}-\left(c_{-x y}+c_{-y x}\right) u_{i-1, j-1, k} \\
& -\left(c_{+x z}+c_{+z x}\right) u_{i+1, j, k+1}+\left(c_{-x z}+c_{+z x}\right) u_{i-1, j, k+1} \\
& +\left(c_{+x z}+c_{-z x}\right) u_{i+1, j, k-1}-\left(c_{-x z}+c_{-z x}\right) u_{i-1, j, k-1} \\
& -\left(c_{+y z}+c_{+z y}\right) u_{i, j+1, k+1}+\left(c_{-y z}+c_{+z y}\right) u_{i, j-1, k+1} \\
& +\left(c_{+y z}+c_{-z y}\right) u_{i, j+1, k-1}-\left(c_{-y z}+c_{-z y}\right) u_{i, j-1, k-1}
\end{aligned}
$$

The expression above is further simplified if $\phi$ is a signed distance function. 


\section{C.2 An additional extension term}

We propose to combine the traditional intrinsic heat flow with an extension term propagating information from the zero level set of $\phi$ to its neighboring levels:

$$
\frac{\partial u}{\partial t}+\operatorname{sign}(\phi)(\nabla u \cdot \nabla \phi)=\Delta_{\Gamma} u .
$$

Note that the flow on the zero level set of $\phi$ remains unchanged. In preliminary experiments, we have found this variant to be numerically advantageous. Indeed, the extension term tends to counteract the numerical diffusion between the zero level set and neighboring levels and to avoid border instabilities when using a narrow band approach. 


\section{References}

[1] S. Angenent, S. Haker, A. Tannenbaum, and R. Kikinis. On area preserving mappings of minimal distorsion. Preprint.

[2] S. Angenent, S. Haker, A. Tannenbaum, and R. Kikinis. Laplace-Beltrami operator and brain surface flattening. IEEE Transactions on Medical Imaging, 18:700-711, 1999.

[3] Richard Barret, Michael Berry, Tony F. Chan, James Demmel, June Donato, Jack Dongarra, Victor Eijkhout, Roldan Pozo, Charles Romine, and Henk van der Vonst. Templates for the Solution of Linear Systems: Building Blocks for Iterative Methods. SIAM, Philadelphia, 1994. Available from netlib.

[4] M. Bertalmio, L.T. Cheng, S. Osher, and G. Sapiro. Variational problems and partial differential equations on implicit surfaces. Journal of Computational Physics, 174:759780, December 2001.

[5] M. Bertalmio, G. Sapiro, and G. Randall. Region Tracking on Surfaces Deforming via Level-Sets Methods. In Mads Nielsen, P. Johansen, O.F. Olsen, and J. Weickert, editors, Scale-Space Theories in Computer Vision, volume 1682 of Lecture Notes in Computer Science, pages 58-69. Springer, September 1999.

[6] Christophe Chefd'hotel, Gerardo Hermosillo, and Olivier Faugeras. Flows of Diffeomorphisms for Multimodal Image Registration. In International Symposium on Biomedical Imaging. IEEE, 2002.

[7] M.C. Delfour and J.-P. Zolésio. Intrinsic differential geometry and theory of thin shells. Quaderni, Scuola Normale Superiore, (Pisa, Italy) to appear, 2000.

[8] M. P. DoCarmo. Differential Geometry of Curves and Surfaces. Prentice-Hall, 1976.

[9] P. Dupuis, U. Grenander, and M. Miller. Variational problems on flows of diffeomorphisms for image matching. Quarterly of Applied Math., 1998.

[10] L.C. Evans. Partial Differential Equations, volume 19 of Graduate Studies in Mathematics. Proceedings of the American Mathematical Society, 1998.

[11] X. Han, C. Xu, and J.L. Prince. A topology preserving level set method for geometric deformable models. IEEE Transactions on Pattern Analysis and Machine Intelligence, 25(6):755-768, June 2003.

[12] G. Hermosillo, O. Faugeras, and J. Gomes. Unfolding the cerebral cortex using level set methods. In Mads Nielsen, P. Johansen, O.F. Olsen, and J. Weickert, editors, Scale-Space Theories in Computer Vision, volume 1682 of Lecture Notes in Computer Science, pages 58-69. Springer, September 1999. 
[13] G.-S. Jiang and D. Peng. Weighted ENO schemes for Hamilton-Jacobi equations. SIAM Journal of Scientific Computing, 21(6):2126-2143, 2000.

[14] G.-S. Jiang and C.-W. Shu. Efficient implementation of weighted ENO schemes. Journal of Computational Physics, 126:202-228, 1996.

[15] R. LeVeque. High-resolution conservative algorithms for advection in incompressible flow. SIAM Journal of Numerical Analysis, 33:627-665, 1996.

[16] William E. Lorensen and Harvey E. Cline. Marching cubes: A high resolution 3d surface construction algorithm. In M.C. Stone, editor, Proceedings of the SIGGRAPH, pages 163-169, Anaheim, CA, July 1987. in Computer Graphics, Volume 21, Number 4.

[17] U.F. Mayer and G. Simonett. Self-intersections for the surface diffusion and the volume preserving mean curature flow. Differential Integral Equations, 13:1189-1199, 2000.

[18] K. Mikula and D. Sevcovic. Evolution of plane curves driven by a nonlinear function of curvature and anisotropy. SIAM Journal of Applied Mathematics, 61(5):1473-1501, 2001.

[19] J. Montagnat, H. Delingette, N. Scapel, and N. Ayache. Representation, shape, topology and evolution of deformable surfaces. Application to 3D medical image segmentation. Technical Report 3954, INRIA, 2000.

[20] S. Osher and J. Sethian. Fronts propagating with curvature dependent speed: algorithms based on the Hamilton-Jacobi formulation. Journal of Computational Physics, $79: 12-49,1988$.

[21] Stanley Osher and Ronald P. Fedkiw. Level set methods : overview and recent results. Tutorials on Geometrically Based Motion, IPAM, Ucla, Los Angeles, 2001.

[22] A. Pazy. Semigroups of Linear Operators and Applications to Partial Differential Equations. Springer-Verlag, 1983.

[23] D. Peng, B. Merriman, S. Osher, H. Zhao, and M. Kang. A PDE-based fast local level set method. Journal on Computational Physics, 155(2):410-438, 1999.

[24] J. Rauch. Partial Differential Equations. Springer-Verlag, New York, 1991.

[25] J.A. Sethian. Level Set Methods and Fast Marching Methods: Evolving Interfaces in Computational Geometry, Fluid Mechanics, Computer Vision, and Materials Sciences. Cambridge Monograph on Applied and Computational Mathematics. Cambridge University Press, 1999.

[26] Alain Trouvé. Diffeomorphisms groups and pattern matching in image analysis. International Journal of Computer Vision, 28(3):213-21, 1998. 
[27] B.C. Vemuri, J. Ye, Y. Chen, and Leonard C.M. A level-set based approach to image registration. In IEEE Workshop on Mathematical Methods in Biomedical Image Analysis, pages 86-93, Hilton Head, South Carolina, June 2000.

[28] J. Xu and H. Zhao. An Eulerian formulation for solving partial differential equations along a moving interface. Technical Report 02-27, UCLA Computational and Applied Mathematics Reports, May 2002.

$\mathrm{RR} \mathrm{n}^{\circ} 4857$ 


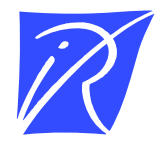

\section{Unité de recherche INRIA Sophia Antipolis 2004, route des Lucioles - BP 93 - 06902 Sophia Antipolis Cedex (France)}

Unité de recherche INRIA Futurs : Parc Club Orsay Université - ZAC des Vignes 4, rue Jacques Monod - 91893 ORSAY Cedex (France)

Unité de recherche INRIA Lorraine : LORIA, Technopôle de Nancy-Brabois - Campus scientifique 615, rue du Jardin Botanique - BP 101 - 54602 Villers-lès-Nancy Cedex (France)

Unité de recherche INRIA Rennes : IRISA, Campus universitaire de Beaulieu - 35042 Rennes Cedex (France)

Unité de recherche INRIA Rhône-Alpes : 655, avenue de l'Europe - 38334 Montbonnot Saint-Ismier (France)

Unité de recherche INRIA Rocquencourt : Domaine de Voluceau - Rocquencourt - BP 105 - 78153 Le Chesnay Cedex (France)

INRIA - Domaine de Voluceau - Rocquencourt, BP 105 - 78153 Le Chesnay Cedex (France)

http://www.inria.fr

ISSN 0249-6399 